\title{
A versatile DNA origami based plasmonic nanoantenna for label-free
}

\section{single-molecule SERS}

\author{
Kosti Tapio ${ }^{1}$, Amr Mostafa $^{1}$, Yuya Kanehira ${ }^{1}$, Antonio Suma ${ }^{2,3}$, Anushree Dutta $^{1}$, Ilko Bald ${ }^{1 *}$ \\ ${ }^{1}$ Institute of Chemistry, University of Potsdam, Potsdam, Germany \\ ${ }^{2}$ Institute for Computational Molecular Science, Temple University, Philadelphia, Pennsy/vania, USA \\ ${ }^{3}$ Dipartimento di Fisica, Università di Bari, and Sezione INFN di Bari, Bari, Italy \\ *corresponding author: ilko.bald@uni-potsdam.de
}

\begin{abstract}
DNA origami technology allows for the precise nanoscale assembly of chemical entities that give rise to new functional materials. We have created a versatile DNA Origami Nanofork Antenna (DONA) by assembling Au or Ag nanoparticle dimers with $1.17 \pm 0.67 \mathrm{~nm}$ gap size, enabling signal enhancements in surface-enhanced Raman scattering (SERS) of up to $10^{11}$. This allows for single-molecule SERS measurements, which can even be performed with larger gap sizes to accommodate differently sized molecules, and at various excitation wavelengths. A general scheme is presented to place single analyte molecules into the SERS hot spots using the DNA origami structure exploiting covalent and non-covalent coupling schemes. By using Au and Ag dimers, single-molecule SERS measurements of three dyes and cytochrome $\mathrm{c}$ and horseradish peroxidase proteins are demonstrated even under nonresonant excitation conditions, thus providing long photostability during time-series measurement, and enabling unprecedented optical monitoring of single molecules and DNA origami based nanomachines.
\end{abstract}


The ultimate detection limit in analytical chemistry is the single molecule (SM), ideally without the necessity to label the target molecule. Furthermore, the ability to extract specific information about single molecular events allows for the screening of molecular property distributions, which are otherwise hidden within an ensemble averaged measurement. ${ }^{1}$ Up to now, several techniques have been reported that are capable of detecting molecules down to the SM regime, such as fluorescence spectroscopy, ${ }^{2}$ scanning probe microscopy, ${ }^{3}$ and surface enhanced Raman scattering (SERS). ${ }^{4}$ Among them, SERS is a particularly promising technique because in addition to the simple detection of a target molecule, it can also provide detailed chemical and structural information through the Raman vibrational fingerprint of the molecule. However, SERS relies on the strong Raman signal enhancement upon excitation of localised surface plasmon resonances (LSPR), mainly in gold ( $\mathrm{Au}$ ) or silver (Ag) nanostructures. ${ }^{5,6}$ The Raman signal enhancement is the strongest in small, nanometer-sized volumes in-between coupled Au or Ag nanostructures, which are referred to as SERS hot spots. Therefore, the detection of single molecules in these hot spots requires a precise control over the arrangement of nanoparticles as well as the placement of target molecules into these hot spots, which imposes severe challenges for nanofabrication. This is why most of the single-molecule SERS measurements reported so far rely on the random adsorption of analyte molecules in the hot spots. ${ }^{4,7,8}$ In a complementary approach the analyte molecules are precisely positioned into the hot spots via covalent or noncovalent interactions using DNA. ${ }^{9}$ DNA origami is a powerful nanoengineering tool, ${ }^{10}$ which is based on the programmed self-assembly into various 2D and 3D shapes. ${ }^{11,12}$ DNA origami nanostructures are formed from a long single-stranded scaffold strand and a suitable set of short artificial staple strands. ${ }^{10}$ Due to the rich chemical functionalization schemes of DNA, molecules can be positioned along the DNA origami surface with nanometer precision. ${ }^{13}$ Furthermore, various kinds of nanoparticles can be arranged on the nanoscale to create sophisticated plasmonic nanostructures and to perform SERS measurements. ${ }^{14-18}$ However, so far the DNA origami based architectures made for SERS measurements relied either on the random adsorption of Raman reporter molecules from solution or were limited to dyes or labelled molecules. ${ }^{15,17,19,20} \mathrm{Here}$, we introduce a new and versatile threedimensional (3D) plasmonic DNA Origami Nanofork Antenna (DONA) structure representing Au and Ag 
dimers suitable for SM SERS measurements. The addressable binding sites in the as-designed DONA structures allow for precise placement of basically arbitrary molecules of different dimension in the gap between two Au or Ag nanoparticles, where the electric field enhancement is strong enough to detect single molecules by SERS.

\section{Results and Discussion}

To create complex arrangements of $\mathrm{Au}$ and $\mathrm{Ag}$ nanoparticles on DNA origami nanostructures the particles are coated with thiolated single-stranded DNA that is hybridized to complementary DNA extensions protruding from the DNA origami. ${ }^{16,21,22} \mathrm{~A}$ frequently used nanoparticle configuration for SERS is the nanoparticle dimer. Importantly, even a small offset from the hot spot can lead to a drastic drop in the field enhancement, which calls for precise positioning of molecules in-between the nanoparticles, which however, has been challenging. To overcome this, we have designed a DNA origami nanofork structure forming the basis of the plasmonic DONA structures. It consists of a rectangular base with two arms, as is illustrated in Fig. 1a and Fig. S1 (Supplementary Information, SI). Two nanoparticles can be bound to the arms such that each particle is connected to both arms. The two arms are connected by a bridge consisting of two DNA double helices. The main purpose of the bridge is to serve as an anchor point for target analyte molecules, which can be precisely placed into the plasmonic gap of the two nanoparticles, at the location where the SERS signal enhancement is the strongest.

The outer dimensions of the DNA origami nanofork are estimated to be $48 \mathrm{~nm} \times 71 \mathrm{~nm}$, based on an inter-helical distance of $2.536 \mathrm{~nm}$ for a honeycomb lattice..$^{23}$ The distance between the two arms is designed to be about $31 \mathrm{~nm}$. Each arm is equipped with four ssDNA capture strands (capture sequences $\mathbf{a}=5^{\prime}-A_{24}$ and $\mathbf{b}=3^{\prime}-(A A C)_{8}$ in Fig. 1 and Fig. S1, to bind two differently coated nanoparticles), extending to different sides and designed to bind complementary sequences on Au or Ag nanoparticles. The bridge includes four staple strands, each crossing over once between the two helices to increase the 
stability and rigidity of the bridge. In addition, the free $3^{\prime}$ and $5^{\prime}$ ends of these staple strands allow for modifications to be added to the bridge. Further, to increase the precision of the nanoparticle placement, two of the bridge staple strands are equipped with capture strands on each end. Hence, each nanoparticle will be bound by a total of six capture strands.
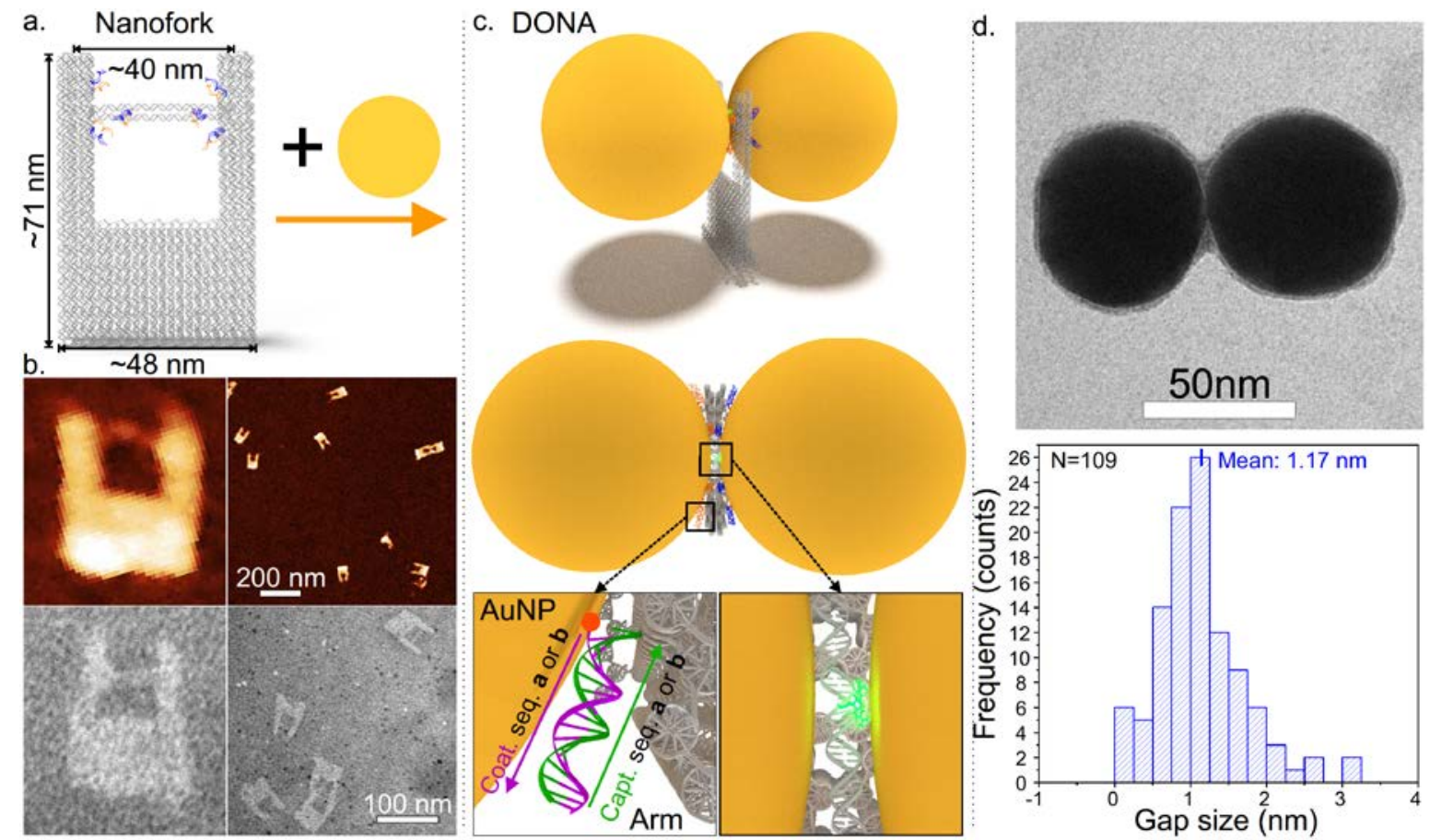

Fig. 1 | Scheme and characterization of the DONA structures. a, Schematic representation of the DNA origami nanofork having a DNA bridge that is $90 \mathrm{nt}$ long. $\mathbf{b}$, AFM and TEM images of the DNA origami nanofork. The sizes of the close-up AFM and TEM images on the lower left are both approx. $90 \mathrm{~nm} \times 100 \mathrm{~nm}$. c, Two differently coated nanoparticles can be attached selectively via DNA hybridization to the two different sequences of DNA capture strands on the arms and the bridge of the DNA origami to form DONA structures. A side-view and a top-view scheme of the assembled DONA structures is shown. The position of an analyte molecule attached to the DNA bridge is illustrated as a green glow. The DNA hybridisation of nanoparticles and DNA origami in the "zipper" configuration is shown in the insets. $d$, Representative TEM image of an assembled Au DONA, along with a distribution of gap sizes determined from TEM images of 109 structures (more examples shown in Fig. S6) with an average gap size of $1.17 \pm 0.67 \mathrm{~nm}$. 
The nanoforks are characterized by atomic force microscopy (AFM) and transmission electron microscopy (TEM) as shown in Fig. 1b. The images show clearly the successful formation of the nanoforks including the DNA bridge. In the zoomed-in AFM image the lower part of the base of the DNA origami fork appears elevated, which corresponds to the additional rows of DNA double helices that are also shown in the scheme of the DNA origami structure. The nanoforks are then hybridized with $\mathrm{Au}$ or Ag nanoparticles of $60 \mathrm{~nm}$ diameter to form the DONAs (Fig. 1c). For optimal SERS measurements a narrow gap between the nanoparticles and precise placement of target molecule therein is required to establish a strong SERS signal enhancement. We have measured the gap distance of the Au DONA structures by TEM, and a typical TEM image is shown in Fig. 1d, along with a histogram of gap sizes. The most probable gap distance is found to be $1.17 \mathrm{~nm}$.

In order to further analyse and optimize the nanofork structure we have performed molecular dynamics simulations using the coarse-grained model oxDNA2. ${ }^{24,25}$ The oxDNA model is ideally suited to analyse the effect of bridge length on the distance of the two DNA arms and the general displacement and fluctuation of the DNA bridge, which is important for the precision of the placement of target molecules. We simulated the nanofork with bridge lengths of 120 nucleotides (nt), $90 \mathrm{nt}$ as well as no bridge. Fig. 2a shows sides and top views of an equilibrated conformation of the DNA origami fork model with a $90 \mathrm{nt}$ bridge, which confirms the overall rigidity of the structure. Fig. $2 \mathrm{~b}$ shows the probability distributions of the distance between the tips of the two DNA arms (dotted lines). The probability distribution is very broad when no bridge is present, with a maximum around $35 \mathrm{~nm}$, which is slightly shorter than the nominal distance of $40 \mathrm{~nm}$ shown in Fig. 1a. With a long bridge of $120 \mathrm{nt}$, the maximum of the probability function is shifted to $45 \mathrm{~nm}$. With a shorter bridge of $90 \mathrm{nt}$, the distribution gets narrower with a most probable distance of $40 \mathrm{~nm}$, corresponding to the designed value. An analysis of AFM images of corresponding nanofork samples yields results that match the simulations (Fig. 2b, solid lines, and Fig. S5 and S6). For further experiments we have chosen a bridge length of $90 \mathrm{nt}$. The simulations also allow to estimate the thermal variation of the centre of the bridge along the plane of the nanofork (Fig. 2c). The plot shows that a shorter DNA bridge increases the 
stiffness of the bridge, and that for the $90 \mathrm{nt}$ bridge a full-width at half maximum (FWHM) of $3.14 \mathrm{~nm}$ is found, indicating the precision with which target molecules placed in the bridge could be located at the plasmonic junction.

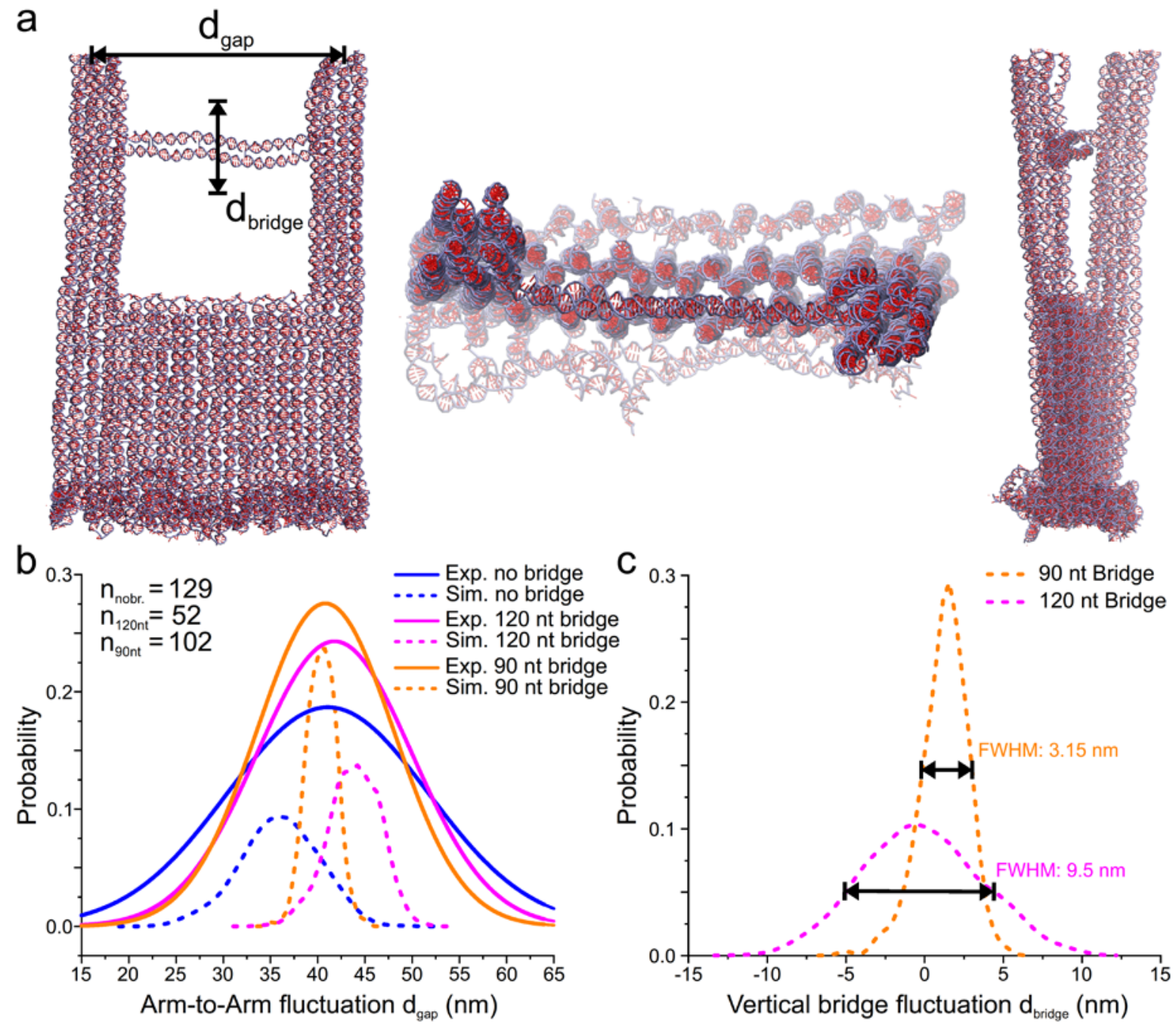

Fig. 2 | Coarse-grained oxDNA model of the DNA origami nanofork. a, Snapshots of two side views and one top-view of the nanofork with 90 nt DNA bridge as simulated using the oxDNA model. $\mathbf{b}$, Plot of the simulated probability distribution of distance between the tips of the two arms and comparison with experimental values obtained from AFM images (for no bridge, $90 \mathrm{nt}$ and $120 \mathrm{nt}$ bridge, the maxima are $41.13 \mathrm{~nm}, 40.82 \mathrm{~nm}$ and $41.84 \mathrm{~nm}$, respectively). The number of nanoforks analysed by AFM is given in the inset. c, Plot of the simulated probability distribution of the flexibility of the centre of a $90 \mathrm{nt}$ and a $120 \mathrm{nt}$ DNA bridge in respect to the straight bridge position, with the maxima at $1.56 \mathrm{~nm}$ and $-0.6 \mathrm{~nm}$, respectively. 
To test the performance of the DONAs for SM SERS we have placed single dye molecules (TAMRA, Cy3.5 and Cy5) at the centre of the DNA bridge using dye-modified staple strands and then assembled $\mathrm{Au}$ and Ag DONAs (see Methods and Table S2). In general, to achieve single molecular sensitivity, a system should produce electric $(E)$ field enhancements in the order of $10^{9}-10^{12}$. To investigate this, we calculated the field enhancement (FE) in the gap between the particles with $60 \mathrm{~nm}$ diameter as a function of wavelength using the finite difference time domain (FDTD) method (see Methods and SI for details). We have considered different gap sizes ranging from $1.2 \mathrm{~nm}$ (corresponding to the mean gap size found in TEM images) to $3.5 \mathrm{~nm}$ as shown in Fig. 3a for Au and in Fig. 3b for Ag dimers. The data in Fig. 3a and 3b allow for an analysis of the SERS signal enhancement of nanoparticle dimers with different gap sizes at specific Raman excitation wavelengths and in respect to the most common excitation lines used in Raman spectroscopy (i.e. $785 \mathrm{~nm}, 633 \mathrm{~nm}, 532 \mathrm{~nm}$ and $488 \mathrm{~nm}$ ). Although the highest FE is predicted for the narrowest gap size $(1.2 \mathrm{~nm})$ around $736 \mathrm{~nm}$ for the Au dimer the FDTD simulations indicate that for Au dimers an excitation at $633 \mathrm{~nm}$ is advantageous because dimers with different gap sizes give rise to a SERS signal enhancement of $10^{9}$ or higher. For example, the FE at 633 $\mathrm{nm}$ excitation wavelength is 255 for a $1.2 \mathrm{~nm}$ gap and 312 for a $2.5 \mathrm{~nm}$ gap, corresponding to a SERS signal enhancement of around $4.2 \times 10^{9}$ and $9.5 \times 10^{9}$, respectively, using the $E^{4}$ approximation. ${ }^{26}$

Besides the plasmonic enhancement, the SERS signal depends also on the optical properties of the analyte molecules, i.e. their optical absorption. The SERS signal can either be further enhanced by the resonance Raman effect by matching the molecule's optical absorption with the $\mathrm{LSPR}^{27}$ or nonresonant molecule excitation can be used to avoid photobleaching.

Fig. 3c shows examples of SM SERS spectra of TAMRA, Cy3.5 and Cy5, which are obtained from Au DONAs excited at $633 \mathrm{~nm}$ and Ag dimers excited at $532 \mathrm{~nm}$ (see Methods and Fig. S11-S14). The black spectra (Fig. 3c) are reference spectra of the respective dyes (see Methods, Fig. S2 and S3 for SERS spectra and S4 for the absorption spectra). Excitation at $532 \mathrm{~nm}$ as used for Ag dimers results in an SM SERS signal with considerably higher signal-to-noise ration than in previous SM SERS studies. ${ }^{15}$ Interestingly, when using $633 \mathrm{~nm}$ excitation for the corresponding Au DONA, SM SERS of TAMRA can 
be measured, although TAMRA does not absorb at $633 \mathrm{~nm}$ and therefore resonant Raman contributions can be excluded in this case. This indicates that the plasmonic field enhancement at 633 $\mathrm{nm}$ is indeed strong enough to detect a single molecule under non-resonant conditions, which is also supported by our FDTD simulations. The same behaviour is observed for Cy3.5, which can be excited by the $532 \mathrm{~nm}$ laser, but not sufficiently by the $633 \mathrm{~nm}$ laser. Nevertheless, strong and characteristic single-molecule SERS spectra are obtained for both, Au and Ag DONAs. The strongest absorption of Cy5 is observed at around $650 \mathrm{~nm}$ (Fig. S4), close to the $633 \mathrm{~nm}$ excitation used for the Au dimers. Consequently, a strong and characteristic single molecule SERS spectrum ${ }^{28}$ is obtained for Cy 5 using the Au DONAs. In contrast, DONAs containing a pure DNA bridge do not display any fingerprint spectra (see Fig. S9 and S10). Therefore, the success of SM SERS measurements using different dyes, nanoparticle species and excitation wavelengths demonstrate the strength and versatility of DONAs, i.e. the ability to tune the plasmonic properties according to the needs of the molecular systems to be investigated. 

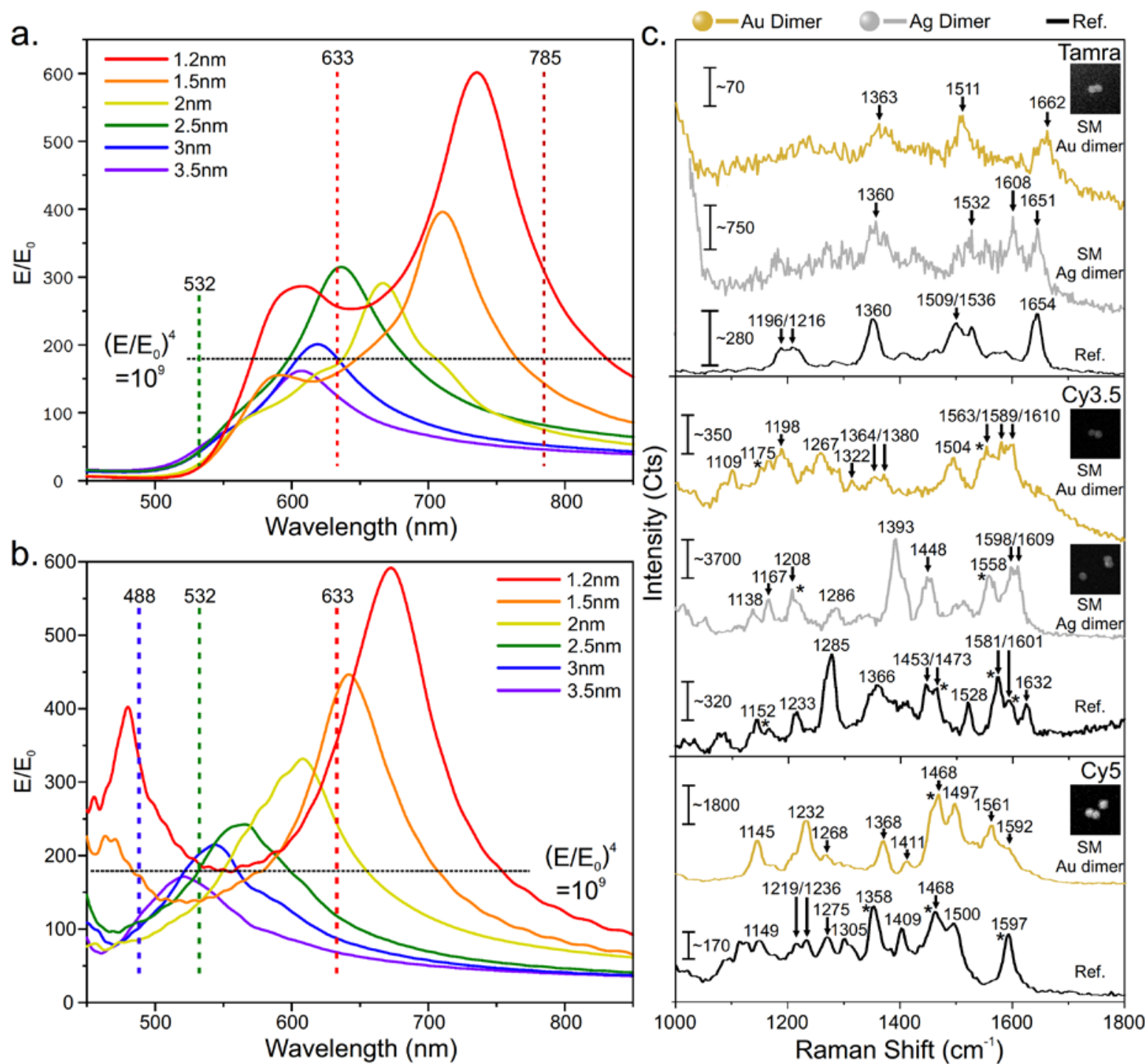

Fig. 3 | FDTD simulations of DNA coated Au and Ag dimers and single-molecule SERS spectra of different dyes. a, FDTD simulations of the E-field enhancement of a Au dimer consisting of DNA coated $60 \mathrm{~nm}$ diameter particles on a silicon substrate. $\mathbf{b}$, FDTD simulations of the E-field enhancement of a DNA coated Ag dimer consisting of $60 \mathrm{~nm}$ diameter particles on a silicon substrate. c, SM SERS spectra of TAMRA, Cy3.5 and Cy5 obtained using Au and Ag DONAs (Cy5 was measured only using Au DONAs). The inset shows SEM images of the specific DONAs, from which the SM SERS spectra have been obtained. The size of the inset SEM images is $450 \times 450 \mathrm{~nm}$. 
Typically, SM SERS spectra show strong fluctuations of the signal intensity with time. Fig. 4 presents a continuous time series SERS measurement of a Au DONA with a TAMRA dye at $633 \mathrm{~nm}$ excitation over the course of 120 seconds recorded using combined dark field and Raman microscopy (see Methods and Fig. S16 and S17). The characteristic signals of TAMRA at $1654 \mathrm{~cm}^{-1}, 1537 \mathrm{~cm}^{-1}$ and $1509 \mathrm{~cm}^{-1} \mathrm{can}$ be clearly identified, while additional signals attributed to DNA are also observed. The characteristic Raman bands can be observed over several minutes, indicating that no photobleaching of the dye occurs, which is attributed to the fact that TAMRA does not absorb the $633 \mathrm{~nm}$ light of the excitation laser. The signal intensity steadily decreases over a time course of several minutes, while the reference signal (Si at $520 \mathrm{~cm}^{-1}$ ) remains rather constant (Fig. S18). This effect could be due to a soldering of the nanoparticles during the constant laser excitation ${ }^{28}$. On a shorter time scale (as displayed in Fig.4), clear fluctuations of the signals are observed, which correspond to SERS blinking. ${ }^{4,29-31}$ One example are the bands at 1537 and $1509 \mathrm{~cm}^{-1}$ : For the first $70 \mathrm{~s}$, the band at $1537 \mathrm{~cm}^{-1}$ is clearly stronger than the band at $1509 \mathrm{~cm}^{-1}$, while the band at $1537 \mathrm{~cm}^{-1}$ almost disappears between $70 \mathrm{~s}$ and $100 \mathrm{~s}$, and subsequently the original intensity ratio is restored. Both bands are assigned to $\mathrm{C}-\mathrm{C}$ stretching vibrations, ${ }^{32}$ the intensity of which varies according to thermal movements of the molecule within the SERS hot spot, giving rise to SERS blinking. Furthermore, it should be noted, that the band expected at 
$1360 \mathrm{~cm}^{-1}$ for TAMRA appears either very weakly during the time series measurements, or it is shifted to $1347 \mathrm{~cm}^{-1}$, e.g. due to the vibrational Stark effect. ${ }^{33}$

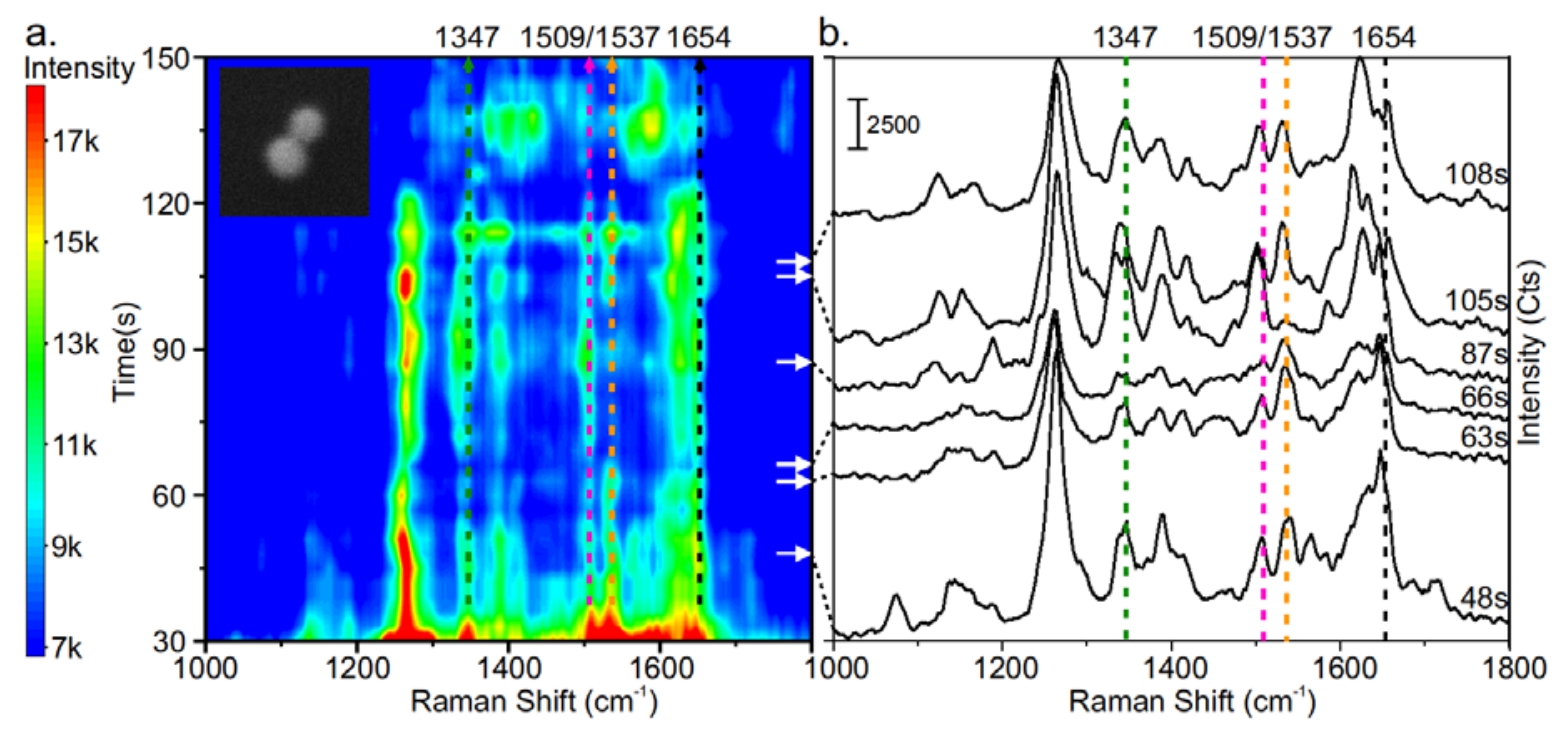

Fig. 4 | Single-molecule SERS time series measurements of TAMRA. a, Time vs Raman shift contour plot for a single TAMRA molecule. The dotted lines indicate the main TAMRA peaks $(1654,1537,1509)$. The dotted green line indicates a band at 1347, which can be either a shifted TAMRA band or a DNA peak. The white arrows indicate different times at which the SERS spectra shown in Fig. $4 \mathrm{~b}$ are extracted. The size of the inset image is $500 \mathrm{~nm} \times 500 \mathrm{~nm}$. b, Full single TAMRA spectra extracted from the contour plot at 48, 63, 66, 87, 105 and $108 \mathrm{~s}$. The same TAMRA bands are indicated with lines as in a.

After demonstrating the potential of DONAs to measure single dye molecules even under nonresonant conditions, we further extend our study to highlight the whole potential of DONAs to directly measure single protein molecules by SERS. This can be achieved with different coupling schemes exploiting either non-covalent or covalent binding of proteins. Here, we have used the heme proteins cytochrome c (cyt c) and horseradish peroxidase (HRP) as model systems to demonstrate their direct, label-free and non-resonant single-molecule SERS detection using DONAs (Fig. 5). Cyt c is found in the inner membranes of mitochondria and is involved in biological electron transfer processes, while HRP 
is involved in oxidation processes. ${ }^{34,35}$ Heme proteins strongly absorb at around $420 \mathrm{~nm}$ (Soret or B band) and $550 \mathrm{~nm}$ ( $Q$ band), ${ }^{36}$ however, we use the $633 \mathrm{~nm}$ excitation laser for the single molecule measurements, which is non-resonant with the heme proteins. In this way, not only the heme prosthetic group is visible in the spectra, but also the amino acid part of the proteins can be directly detected.
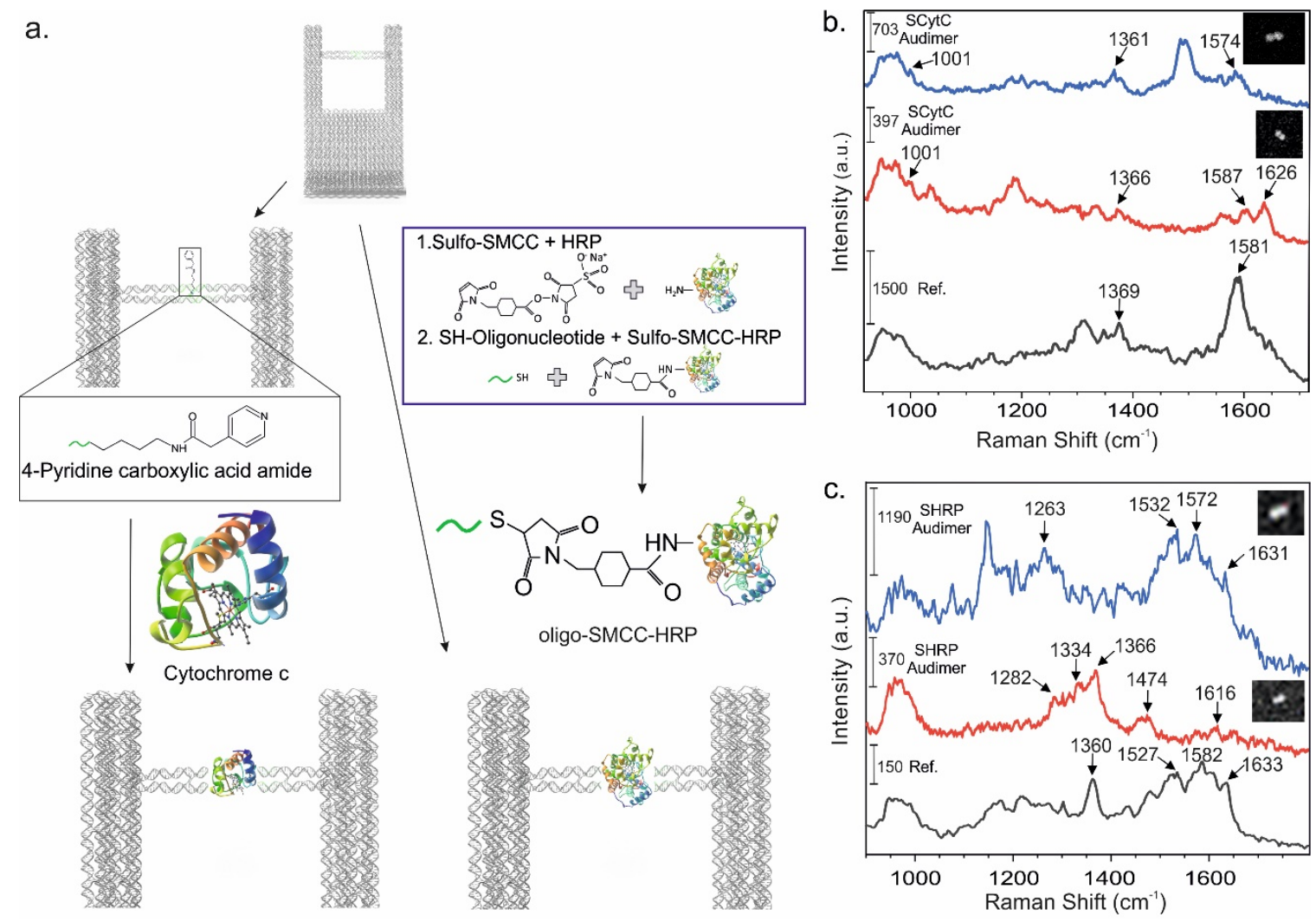

Fig. 5 | Single-molecule SERS measurements of cytochrome $c$ (cyt c) and horseradish peroxidase (HRP). a, Scheme of the non-covalent and covalent coupling schemes to bind cyt $\mathrm{c}$ and HRP to the DNA bridge of the nanofork. Cyt c is coupled non-covalently to pyridine, which is connected to the DNA bridge, while HRP is cross-linked to a DNA staple strand, which is part of the DNA bridge. b, SM SERS spectra of cyt c using Au DONA structures and $633 \mathrm{~nm}$ excitation. c, SM SERS spectra of HRP using Au DONA structures and $633 \mathrm{~nm}$ excitation. The insets in $b$ and $c$ show SEM images of the specific DONAs, from which the SM SERS spectra have been obtained. The sizes of the SEM images are from top to bottom $420 \times 345 \mathrm{~nm}, 360 \times 360 \mathrm{~nm}, 240 \times$ $240 \mathrm{~nm}$ and $480 \times 380 \mathrm{~nm}$, respectively. 
Fig. 5a shows the binding schemes of cyt $\mathrm{c}$ and HRP to the nanofork. For the non-covalent, but specific binding of cyt c to the DONAs, a 4-pyridine moiety ${ }^{37}$ is coupled via an amide bond and a C5 linker to a staple strand at the centre of the DNA bridge (the sequence shown in Table S2). HRP is covalently coupled to the NHS ester group of a Sulfo-SMCC cross linker via a lysine residue. The maleimide group of the cross linker is then bound to a thiolated staple strand (Table S2), which in turn is incorporated into the DNA bridge of the DNA origami nanofork. Both, cyt $\mathrm{c}$ and HRP are bound to the DNA origami nanofork prior to the binding of the $60 \mathrm{~nm}$ Au nanoparticles. Fig. $5 \mathrm{~b}$ and $5 \mathrm{c}$ show the single-molecule SERS spectra of cyt c and HRP (blue and red spectra), respectively, recorded at $633 \mathrm{~nm}$ excitation along with the corresponding SEM images of Au dimers and a reference spectrum (black spectra). The reference spectra were recorded for cyt c or HRP, respectively, unspecifically bound to clusters of 60 $\mathrm{nm}$ Au nanoparticles (see Methods). The SERS spectrum of a single cyt c molecule recorded with a Au DONA matches with the reference spectrum. The characteristic band appearing in the range 1361 $1369 \mathrm{~cm}^{-1}$ is assigned to the stretching vibration of the porphyrin ring $\left(v_{4}\right)$ of the heme group indicating the oxidation state $\left(\mathrm{Fe}^{2+}\right)$ of the central iron ion. ${ }^{36,38}$ The strong signal around $1580 \mathrm{~cm}^{-1}$ is assigned to the $v_{19}$ band. ${ }^{36}$ Apart from the signals arising from the heme unit, the vibrational ring-breathing mode of the amino acid phenylalanine (Phe) can also be clearly distinguished at $1001 \mathrm{~cm}^{-1} \cdot{ }^{39} \mathrm{Cyt}$ c possesses four Phe residues, and the possibility to detect these in a single cyt c molecule by SERS using DONAs indicates the high sensitivity of the method.

Fig. 5c shows SM SERS spectra of HRP coupled to Au DONAs along with a reference spectrum (black). SERS signals characteristic for HRP can be clearly detected, such as the oxidation state marker at 1633 $\mathrm{cm}^{-1}\left(\mathrm{v}_{10}\right)$, indicating the presence of $\mathrm{Fe}^{3+} \cdot{ }^{36}$ To the best of our knowledge, the present data are the first reports of SM SERS spectra of HRP under non-resonant conditions.

In conclusion, the newly designed 3D DNA origami nanofork has successfully been demonstrated for its suitability to assemble both nanoparticles and complex analyte molecules with nanoscale precision for SM SERS measurements. OxDNA simulations were employed to assess the structural dynamics of 
the nanofork and to choose the most suitable bridge length. The DNA bridge connecting the two arms of the nanofork allows for precise positioning of the target analyte in between the assembled nanoparticles of $\mathrm{Au}$ and $\mathrm{Ag}$. This produces SERS hot spots in the scale of few nanometers, creating high enough field enhancement to enable label-free detection of single small molecules and even protein (cyt c and HRP) via SERS, even at larger gap sizes around $2.5 \mathrm{~nm}$. Importantly, the flexible integration of either Au and Ag nanoparticles supports SM SERS measurements both in resonant and non-resonant conditions. Time-dependent SM SERS of TAMRA under non-resonant conditions shows spectral blinking but no significant photobleaching, strikingly reflecting the single-molecular SERS behaviour. The experimental findings are supported by FDTD simulations that predict field enhancements high enough for single molecule measurements under non-resonant conditions. DNA origami is a remarkable technique that grants control over single molecular entities, thereby enabling novel types of nanomachines. ${ }^{13,40}$ Here, we used this principle to build a platform for the direct, chemically-specific and label-free optical detection of single small molecules and single proteins. This opens new potential to study biomolecular reactions at the single molecule level. The DONA structures could be used to monitor enzymatic action in different environments, perhaps even for multi-enzyme reaction cascades. At the same time, the sensing of single small molecules represents one of the greatest challenges in analytical chemistry. Through the study of single reaction intermediates it could prove invaluable for understanding chemical reaction pathways.

\section{Methods}

\section{DNA origami nanofork folding and purifications}

The staple strands used for the assembly of the DNA origami nanofork are shown in Table S1. Depending on the experiment, some strands were substituted with the modified versions of the same strand (see Table S2). The folding solution was made by mixing $10 \mu \mathrm{l}$ of $10 \mathrm{x}$ buffer (10xTAE, $150 \mathrm{mM} \mathrm{MgCl}$ ), $67.5 \mu$ l of Millipore water, 20 $\mu \mathrm{l}$ of staple solution (the concentration of individual staple $=0.497 \mu \mathrm{M})$ and $2.5 \mu \mathrm{l}$ of the scaffold $(\mathrm{m} 13 \mathrm{mp} 18,0.1$ $\mu \mathrm{M})$. During folding, the ratio between staples and the scaffold is $40: 1$. The folding solution was heated to $80^{\circ} \mathrm{C}$ 
and then cooled down to $20^{\circ} \mathrm{C}$ over $15 \mathrm{~h}$ timespan. The solution was purified from excess oligos using $100 \mathrm{kDa}$ centrifugal filters (Amicon, Ultracel, Centrifugal filter, $100 \mathrm{kDA}$ ): the DNA origami solution (100 $\mu$ l) was added to the filter and $400 \mu \mathrm{l}$ of Millipore water was mixed in, the filter was spun in $4700 \mathrm{rcf}$ for $5 \mathrm{~min}$, the filtrate was discarded and $450 \mu \mathrm{l}$ of Millipore water was added. The centrifugation was repeated 3 times and the dead volume from the filter was finally recovered. The concentration of $\mathrm{TAE}$ and $\mathrm{MgCl}_{2}$ was adjusted to $1 \mathrm{x}$ and $5 \mathrm{mM}$, respectively. A UV-vis spectrometer was used to measure the DNA concentration of the final solution that was used to calculate the DNA origami concentration.

\section{Nanoparticles coating protocols}

Gold (Au nanosphere NanoXact, citrate coated, $60 \mathrm{~nm}, 2.4 \times 10^{10}$ particles/ml) and silver (Ag nanosphere NanoXact, citrate coated, $60 \mathrm{~nm}, 1.9 \times 10^{10}$ particles/ml) nanoparticles were purchased from nanoComposix (4878 Ronson Ct Ste J, San Diego, USA). The DNA strands in the table S1 were acquired from Sigma Aldrich (SigmaAldrich Chemie GmbH, Munich, Germany). Tris(2-carboxyethyl)phosphine (TCEP) was purchased from RIZ Biochem.

The gold nanoparticles were coated using a modified salt aging method, whereas the coating for silver nanoparticles was done using a pH altering method. ${ }^{41}$ For AuNPs, $400 \mu \mathrm{l}$ of $60 \mathrm{~nm}$ AuNP stock solution were centrifuged once (2900 rcf, $5 \mathrm{~min}$ ), the supernatant was removed and the volume was adjusted to $26.5 \mu \mathrm{l}$ by MilliQ water. The disulphide bonds in the DPTA-modified coating strands were pre-cleaved using TCEP: $4 \mu$ of the coating strand solution $(100 \mu \mathrm{M})$ was mixed with $1 \mu$ l of TCEP $(100 \mathrm{mM})$ and incubated at RT for $10 \mathrm{~min}$. The $5 \mu \mathrm{l}$ of cleaved DNA strand solution and $3.5 \mu \mathrm{l}$ of $0.2 \%$ SDS was added to the AuNP solution and incubated 40 min to $1 \mathrm{~h}$ and at $40{ }^{\circ} \mathrm{C}$. After the incubation, the concentration of $\mathrm{NaBr}$ was gradually, in stepwise manner increased to $300 \mathrm{mM}$ using initially $400 \mathrm{mM} \mathrm{NaBr}$ buffer and then $1 \mathrm{M}$ buffer, where after each step the solution was incubated for $10 \mathrm{~min}$ at $40{ }^{\circ} \mathrm{C}$ : initial 4 steps were $1.7 \mu \mathrm{l}(400 \mathrm{mM})$, the next two $2.1 \mu \mathrm{l}$ and $2.3 \mu \mathrm{l}(400 \mathrm{mM})$, followed by $3 \times 2.3 \mu \mathrm{l}$ and $1 \mathrm{M}$ and finally $6.5 \mu \mathrm{l}$ and $1 \mathrm{M}$. After the $\mathrm{NaBr}$ addition, $1 \mathrm{x}$ TAE and $5 \mathrm{mM} \mathrm{MgCl} 2$ was added twice $(6 \mu \mathrm{l}$ and $6.6 \mu \mathrm{l})$ in similar stepwise manner. Finally, the excess coating strands were purified by centrifugation: the sample was spun down using $2900 \mathrm{rcf}$ and $5 \mathrm{~min}$, supernatant was removed and buffer added. During the first two round of centrifugation, $1 \times T A E, 5 \mathrm{mM} \mathrm{MgCl}_{2}$ and $0.02 \% \mathrm{SDS}$ buffer was used and during the final two rounds, the buffer was switched to 1 XTAE and $5 \mathrm{mM} \mathrm{MgCl}$. 
For silver particles, $400 \mu \mathrm{l}$ of the $60 \mathrm{~nm}$ AgNP solution was centrifuged once ( $2900 \mathrm{rcf}, 5 \mathrm{~min}$ ), the supernatant was removed and the volume was adjusted to $26.5 \mu$ l by MilliQ water. The coating strands were cleaved similarly as in the case of AuNPs using TCEP. $3.5 \mu \mathrm{l}$ of $0.2 \%$ SDS and $5 \mu$ l of DNA solution were added to the AgNP solution and incubated for $40 \mathrm{~min}$ at RT. Then the $\mathrm{pH}$ of the solution was adjusted close to 3 by adding $1.4 \mu$ l of $0.5 \mathrm{M}$ TSC $(\mathrm{pH}$ 3, adjusted using $\mathrm{HCl}$ ) to the mixture. After 3 min incubation at $\mathrm{RT}, \mathrm{NaCl}(1 \mathrm{M})$ was added in stepwise manner until the final $\mathrm{NaCl}$ concentration of the AgNP solution was $0.3 \mathrm{M}: 2 \mu \mathrm{l}$ of $\mathrm{NaCl}$ was added 6 times followed by final addition of $3.6 \mu \mathrm{l}$, where in-between additions the solution was rapidly vortexed and let stand still for few seconds. The particles were incubated for $5 \mathrm{~min}$ after the final addition of the $\mathrm{NaCl}$, and purified similarly as the AuNPs.

DONA assembly, gel electrophoresis purifications

DONAs were fabricated by mixing DNA origami nanoforks with two different $60 \mathrm{~nm}$ AuNP/AgNP batches, where one particle is coated with $\mathrm{T}_{28}$ sequence and the other with $(\mathrm{GTT})_{8} \mathrm{~T}_{4}$ sequence. All materials were dissolved in 1XTAE and $5 \mathrm{mM} \mathrm{MgCl} 2$ buffer. The ratio of NP-T28:NP-(GTT) $)_{8} \mathrm{~T}_{4}:$ nanofork is $1.5: 1.5: 1$ and the final concentration of nanoforks is between 0.1 and $0.2 \mathrm{nM}$. The mixture was heated in a thermocycler to $40{ }^{\circ} \mathrm{C}$ and then cooled down to $20^{\circ} \mathrm{C}$ in $3.5 \mathrm{~h}$ timespan. In some cases such as nanoforks with dyes, agarose gel electrophoresis was used to extract the dimers from the final product. Shortly, samples were run in $1 \%$ agarose gel made in $1 \times T A E$ and $5 \mathrm{mM} \mathrm{MgCl}_{2}$ for 60 min using $80 \mathrm{~V}$. The dimer band was cut out of the gel and the dimers were squeezed out using two microscope slides.

$\underline{\text { TEM imaging of DNA origami nanoforks and DONAs }}$

All samples were imaged in JEOL JEM 1011 transmission electron microscope (JEOL, Akishima, Tokyo, Japan) equipped with Olympus MegaView G2 camera and using $80 \mathrm{kV}$ acceleration voltage. In the TEM imaging, copper grids containing $1 \mathrm{~nm}$ carbon layer on top of $10 \mathrm{~nm}$ formvar film (EFCF400-Cu-50, Science Services GmbH, Unterhachinger Straße 75, Munich, Germany) were used. To characterize the dimer gap sizes, the DONA solution was deposited on a TEM grid. For DONA deposition, no plasma-treatment was required whereas, for nanoforks, the TEM grids were plasma-treated for 15 s using Diener Electronic Zepto plasma cleaner before deposition of the DNA origami and the staining. In the case of DONAs, $3 \mu \mathrm{l}$ of DONA solution was pipetted to the grid and incubated for 2-3 min. The excess solution was blotted away and $3 \mu \mathrm{l}$ of staining solution was added. After 2 min 
incubation, the excess staining solution was blotted away and the film was washed twice by adding $3 \mu$ of Millipore water to the grid and blotting away the excess liquid. The grid was let to dry in ambient conditions.

In the case of DNA origami nanofork, after the plasma treatment, roughly $3 \mu$ of DNA origami solution was pipetted on a grid and incubated for $5 \mathrm{~min}$. The magnesium and fork concentrations were $20 \mathrm{mM}$ and $0.6 \mathrm{nM}$, respectively. The excess solution was blotted away and $3 \mu \mathrm{l}$ of the same staining solution was added. After 2 min incubation, the excess solution was blotted away and the sample was left to dry in ambient condition without any washing. Figure S7 contains extra images of the DNA origami nanoforks.

\section{Sample preparation for SERS, SERS measurements, SEM imaging and SERS-SEM correlation}

DONAs were deposited on a $7 \mathrm{~mm} \times 7 \mathrm{~mm}$ silicon chip labelled with a scratch-marker. A diamond cutter was used to draw a cross-shape on the chip, the surface was scrubbed clean from dust in acetone solution using a cotton stick and finally sonicating the chip in ethanol solution for $30 \mathrm{~s}$. The clean chip was plasma-treated for $10 \mathrm{~min}$. For the deposition, the magnesium concentration of DONA solution was adjusted to $50 \mathrm{mM}$, the solution was pipetted to plasma-treated silicon surface and incubated for roughly $1.5 \mathrm{~h}$. The chip was washed with ethanolwater solution (1:1 ratio) and blown dry.

A Witec Alpha 300 Raman microscope was used in the measurements (Witec, Ulm, Germany). For the 488 and $532 \mathrm{~nm}$ measurements a grating spectrograph (Blaze 500, grating $600 \mathrm{gr} / \mathrm{mm}$ ) equipped with Andor DV401-BV CCD-camera was used, and for the $633 \mathrm{~nm}$ measurements a spectrograph (Blaze 750, grating $600 \mathrm{gr} / \mathrm{mm}$ ) equipped with Andor DU401A-BR-DD-352 CCD-camera. The estimated spot sizes were $1.32 \mu \mathrm{m}, 1.44 \mu \mathrm{m}$ and $1.72 \mu \mathrm{m}$ for $488 \mathrm{~nm}, 532 \mathrm{~nm}$ and $633 \mathrm{~nm}$, respectively. Raman maps were acquired using varying laser power densities and integration times per point (see SI). After a Raman measurement, the Raman map area was imaged using SEM (Thermo Fisher Phenom ProX Desktop SEM or FEI Quanta 250) where the scratch-markers acted as reference points to identify the area scanned with the Raman microscope. For the dye references, particles coated with dye-polyT oligos were deposited on a silicon surface and point spectra were measured using 532 or $633 \mathrm{~nm}$ laser (see SI for more details). For protein samples, the proteins were incubated with the nanoparticles before to adsorb them to the particle surface and cause aggregation. Then the aggregates were deposited on a silicon surface and point measurements were performed using the $633 \mathrm{~nm}$ laser and $300 \mu \mathrm{W}(200 \mu \mathrm{W})$ laser power and $8 \mathrm{~s}(12 \mathrm{~s})$ integration time for cyt c (HRP). 
A detailed overview of the FDTD simulations can be found in the SI. In brief, we used the Lumerical FDTD Solutions software (v8.19.1584). The model included two gold or silver spheres (refractive index from Johnson and Christy) ${ }^{42}$ surrounded by $1.12 \mathrm{~nm}$ DNA layer (refractive index 1.7$)^{43}$ and on top of a silicon substrate (refractive index from ${ }^{44}$ ) with $3 \mathrm{~nm}$ silicon dioxide (refractive index 1.44) on top of the Si (see Fig. S21). The DNA layer thickness was measured using AFM (Figs. S19 and S20). Here, we are omitting the bridge and the DNA origami nanofork from the framework. The medium was defined as air. The distance between gold spheres was varied according to the measured distance from Fig. 1 (from $1.2 \mathrm{~nm}$ to $3.5 \mathrm{~nm}$ ), where a water layer was added between DNA layers in the case of 2.5 and $3 \mathrm{~nm}$ particle-to-particle distances. The E field distributions were calculated and the field maximum around the gap region was recorded. To solve the Eo case, we removed the DNA layers and the metal spheres. Electric field polarizations along the gap (the gap mode) and perpendicular to gap (the offgap mode) were considered. The results show that the electric field is highly localized within the gap region in the case of the gap mode and the off-gap mode excitation results mainly in plasmonic mode that resembled single particle dipole excitation (Figs. S22-S26).

\section{OxDNA simulations}

We performed Langevin molecular dynamics simulations for the three nanofork structures considered (90 nt, $120 \mathrm{nt}$ and no bridge) using the coarse-grained DNA model oxDNA2, ${ }^{24,25}$ parametrized to reproduce mechanical and thermodynamic experimental properties of DNA. The initial oxDNA configurations were obtained converting the caDNAno files with the tacoxDNA package. ${ }^{45}$ Simulations were carried out with the LAMMPS software implementation. ${ }^{46}$ The temperature was set to $\mathrm{T}=300 \mathrm{~K}$ and the monovalent salt concentration to $1 \mathrm{M}$. Each system was simulated for about $2 \times 10^{6}$ simulation time units, for a total of 2000 configurations sampled.

\section{Acknowledgment}

This research was supported by the European Research Council (ERC; consolidator grant no. 772752). We thank Sibylle Rüstig for assisting in TEM imaging.

\section{Author contributions}


IB conceived the study, KT and AM prepared and characterized DONAs, KT did the TEM imaging and dye measurements and performed FDTD simulations, AM did sample preparations and measurement involving cyt c, YK did sample preparations and measurement involving HRP, AS performed OxDNA simulations, and all authors analysed the data and contributed to manuscript writing.

\section{Competing interests}

There are no competing interests.

\section{References:}

1. Claridge, S. A., Schwartz, J. J. \& Weiss, P. S. Electrons, photons, and force: quantitative singlemolecule measurements from physics to biology. ACS Nano 5, 693-729; 10.1021/nn103298x (2011).

2. W. E. Moerner \& David P. Fromm. Methods of single-molecule fluorescence spectroscopy and microscopy. Rev. Sci. Instrum. 74, 3597; 10.1063/1.1589587 (2003).

3. Pavliček, N. \& Gross, L. Generation, manipulation and characterization of molecules by atomic force microscopy. Nat. Rev. Chem. 1; 10.1038/s41570-016-0005 (2017).

4. Zrimsek, A. B. et al. Single-Molecule Chemistry with Surface- and Tip-Enhanced Raman Spectroscopy. Chem. Rev. 117, 7583-7613; 10.1021/acs.chemrev.6b00552 (2017).

5. Pilot, R. et al. A Review on Surface-Enhanced Raman Scattering. Biosensors 9; 10.3390/bios9020057 (2019).

6. Langer, J. et al. Present and Future of Surface-Enhanced Raman Scattering. ACS Nano 14, 28-117; 10.1021/acsnano.9b04224 (2020).

7. Lee, H. M., Jin, S. M., Kim, H. M. \& Suh, Y. D. Single-molecule surface-enhanced Raman spectroscopy: a perspective on the current status. Phys. Chem. Chem. Phys. 15, 5276-5287; 10.1039/c3cp44463e (2013).

8. Le Ru, E. C. \& Etchegoin, P. G. Single-molecule surface-enhanced Raman spectroscopy. Annu. Rev. Phys. Chem. 63, 65-87; 10.1146/annurev-physchem-032511-143757 (2012).

9. Lee, J.-H. et al. Tuning and maximizing the single-molecule surface-enhanced Raman scattering from DNA-tethered nanodumbbells. ACS Nano 6, 9574-9584; 10.1021/nn3028216 (2012).

10. Rothemund, Paul W. K. Folding DNA to create nanoscale shapes and patterns. Nature $\mathbf{4 4 0 , 2 9 7 -}$ 302; 10.1038/nature04586 (2006). 
11. Dietz, H., Douglas, S. M. \& Shih, W. M. Folding DNA into twisted and curved nanoscale shapes. Science 325, 725-730; 10.1126/science.1174251 (2009).

12. Wagenbauer, K. F., Sigl, C. \& Dietz, H. Gigadalton-scale shape-programmable DNA assemblies. Nature 552, 78-83; 10.1038/nature24651 (2017).

13. Tapio, K. \& Bald, I. The potential of DNA origami to build multifunctional materials. Multifunctional Materials (2020).

14. Prinz, J. et al. DNA Origami Substrates for Highly Sensitive Surface-Enhanced Raman Scattering. J. Phys. Chem. Lett. 4, 4140-4145; 10.1021/jz402076b (2013).

15. Prinz, J., Heck, C., Ellerik, L., Merk, V. \& Bald, I. DNA origami based Au-Ag-core-shell nanoparticle dimers with single-molecule SERS sensitivity. Nanoscale 8, 5612-5620; 10.1039/c5nr08674d (2016).

16. Heck, C. et al. Gold Nanolenses Self-Assembled by DNA Origami. ACS Photonics 4, 1123-1130; 10.1021/acsphotonics.6b00946 (2017).

17. Heck, C., Kanehira, Y., Kneipp, J. \& Bald, I. Placement of Single Proteins within the SERS Hot Spots of Self-Assembled Silver Nanolenses. Angew. Chem. Int. Ed. 57, 7444-7447; 10.1002/anie.201801748 (2018).

18. Liu, N. \& Liedl, T. DNA-Assembled Advanced Plasmonic Architectures. Chem. Rev. 118, 30323053; 10.1021/acs.chemrev.7b00225 (2018).

19. Tanwar, S., Haldar, K. K. \& Sen, T. DNA Origami Directed Au Nanostar Dimers for Single-Molecule Surface-Enhanced Raman Scattering. J. Am. Chem. Soc. 139, 17639-17648; 10.1021/jacs.7b10410 (2017).

20. Zhan, P. et al. DNA Origami Directed Assembly of Gold Bowtie Nanoantennas for Single-Molecule Surface-Enhanced Raman Scattering. Angew. Chem. Int. Ed. 57, 2846-2850; 10.1002/anie.201712749 (2018).

21. Ding, B. et al. Gold nanoparticle self-similar chain structure organized by DNA origami. J. Am. Chem. Soc. 132, 3248-3249; 10.1021/ja9101198 (2010).

22. Shen, X. et al. Three-dimensional plasmonic chiral tetramers assembled by DNA origami. Nano Lett. 13, 2128-2133; 10.1021/nl400538y (2013).

23. Fischer, S. et al. Shape and Interhelical Spacing of DNA Origami Nanostructures Studied by SmallAngle X-ray Scattering. Nano Lett. 16, 4282-4287; 10.1021/acs.nanolett.6b01335 (2016).

24. Snodin, B. E. K. et al. Introducing improved structural properties and salt dependence into a coarse-grained model of DNA. J. Chem. Phys. 142, 234901; 10.1063/1.4921957 (2015).

25. Ouldridge, T. E., Louis, A. A. \& Doye, J. P. K. Structural, mechanical, and thermodynamic properties of a coarse-grained DNA model. J. Chem. Phys. 134, 85101; 10.1063/1.3552946 (2011).

26. Ding, S.-Y., You, E.-M., Tian, Z.-Q. \& Moskovits, M. Electromagnetic theories of surface-enhanced Raman spectroscopy. Chem. Soc. Rev. 46, 4042-4076; 10.1039/c7cs00238f (2017).

27. McNay, G., Eustace, D., Smith, W. E., Faulds, K. \& Graham, D. Surface-enhanced Raman scattering (SERS) and surface-enhanced resonance Raman scattering (SERRS): a review of applications. Appl. Spectrosc. 65, 825-837; 10.1366/11-06365 (2011). 
28. Salmon, A. R. et al. Light-Induced Coalescence of Plasmonic Dimers and Clusters. ACS Nano; 10.1021/acsnano.0c01213 (2020).

29. Itoh, T. \& Yamamoto, Y. S. Recent topics on single-molecule fluctuation analysis using blinking in surface-enhanced resonance Raman scattering: clarification by the electromagnetic mechanism. Analyst 141, 5000-5009; 10.1039/c6an00936k (2016).

30. Kitahama, Y., Nagahiro, T., Tanaka, Y., Itoh, T. \& Ozaki, Y. Analysis of blinking from multicoloured SERS-active Ag colloidal nanoaggregates with poly-L-lysine via truncated power law. J. Raman Spectrosc. 48, 570-577; 10.1002/jrs.5079 (2017).

31. Lombardi, J. R., Birke, R. L. \& Haran, G. Single Molecule SERS Spectral Blinking and Vibronic Coupling. J. Phys. Chem. C 115, 4540-4545; 10.1021/jp111345u (2011).

32. Jaworska, A., Pyrak, E. \& Kudelski, A. Comparison of the efficiency of generation of Raman radiation by various Raman reporters connected via DNA linkers to different plasmonic nanostructures. Vib. Spectrosc. 101, 34-39; 10.1016/j.vibspec.2019.01.002 (2019).

33. Marr, J. M. \& Schultz, Z. D. Imaging Electric Fields in SERS and TERS Using the Vibrational Stark Effect. J. Phys. Chem. Lett. 4; 10.1021/jz401551u (2013).

34. Bertini, I., Cavallaro, G. \& Rosato, A. Cytochrome c: occurrence and functions. Chem. Rev. 106, 90-115; 10.1021/cr050241v (2006).

35. Veitch, N. C. Horseradish peroxidase: a modern view of a classic enzyme. Phytochemistry 65, 249-259; 10.1016/j.phytochem.2003.10.022 (2004).

36. Rygula, A. et al. Raman spectroscopy of proteins: a review. J. Raman Spectrosc. 44, 1061-1076; 10.1002/jrs.4335 (2013).

37. Yamamoto, H., Liu, H. \& Waldeck, D. H. Immobilization of cytochrome c at Au electrodes by association of a pyridine terminated SAM and the heme of cytochrome. Chem. Commun., 10321033; 10.1039/b101767p (2001).

38. Kitahama, Y. \& Ozaki, Y. Surface-enhanced resonance Raman scattering of hemoproteins and those in complicated biological systems. Analyst 141, 5020-5036; 10.1039/c6an01009a (2016).

39. Yeo, B.-S., Mädler, S., Schmid, T., Zhang, W. \& Zenobi, R. Tip-Enhanced Raman Spectroscopy Can See More: The Case of Cytochrome c. J. Phys. Chem. C 112, 4867-4873; 10.1021/jp709799m (2008).

40. Ramezani, H. \& Dietz, H. Building machines with DNA molecules. Nat. Rev. Genet. 21, 5-26; 10.1038/s41576-019-0175-6 (2020).

41. Zhang, X., Servos, M. R. \& Liu, J. Instantaneous and quantitative functionalization of gold nanoparticles with thiolated DNA using a pH-assisted and surfactant-free route. J. Am. Chem. Soc. 134, 7266-7269; 10.1021/ja3014055 (2012).

42. Johnson, P. B. \& Christy, R. W. Optical Constants of the Noble Metals. Phys. Rev. B 6, 4370-4379; 10.1103/PhysRevB.6.4370 (1972).

43. Thacker, V. V. et al. DNA origami based assembly of gold nanoparticle dimers for surfaceenhanced Raman scattering. Nat. Commun. 5, 3448; 10.1038/ncomms4448 (2014).

44. Pierce, D. T. \& Spicer, W. E. Electronic Structure of Amorphous Si from Photoemission and Optical Studies. Phys. Rev. B 5, 3017-3029; 10.1103/PhysRevB.5.3017 (1972). 
45. Suma, A. et al. TacoxDNA: A user-friendly web server for simulations of complex DNA structures, from single strands to origami. J. Comput. Chem. 40, 2586-2595; 10.1002/jcc.26029 (2019).

46. Henrich, O., Gutiérrez Fosado, Y. A., Curk, T. \& Ouldridge, T. E. Coarse-grained simulation of DNA using LAMMPS : An implementation of the oxDNA model and its applications. Eur. Phys. J. E 41, 57; 10.1140/epje/i2018-11669-8 (2018). 